\title{
Automated Computer-Based Enumeration of Acellular Capillaries for Assessment of Diabetic Retinopathy
}

\author{
Mihran Tuceryan $^{\mathrm{a}}$, Anish N. Hemmady ${ }^{\mathrm{a}, \mathrm{b}}$, Craig Schebler ${ }^{\mathrm{a}, \mathrm{b}}$, Alpha Alex ${ }^{\mathrm{b}}$, and Ashay D. \\ Bhatwadekar ${ }^{\mathrm{b}}$ \\ ${ }^{a}$ Department of Computer and Information Science, Indiana University-Purdue University \\ Indianapolis, USA \\ ${ }^{\mathrm{b}}$ Department of Ophthalmology, Indiana University, Indianapolis, IN, USA
}

\begin{abstract}
Diabetic retinopathy (DR) is the most common complications of diabetes; if untreated the DR can lead to a vision loss. The treatment options for DR are limited and the development of newer therapies are of considerable interest. Drug screening for the retinopathy treatment is undertaken using animal models in which the quantification of acellular capillaries (capillary without any cells) is used as a marker to assess the severity of retinopathy and the treatment response. The traditional approach to quantitate acellular capillaries is through manual counting. The purpose of this investigation was to develop an automated technique for the quantitation of acellular capillaries using computer-based image processing algorithms. We developed a custom procedure using the Python, the medial axis transform (MAT) and the connected component algorithm. The program was tested on the retinas of wild-type and diabetic mice and the results were compared to single blind manual counts by two independent investigators. The program successfully identified and enumerated acellular capillaries. The acellular capillary counts were comparable to the traditional manual counting. In conclusion, we developed an automated computer-based program, which can be effectively used for future pharmacological development of treatments for DR. This algorithm will enhance consistency in retinopathy assessment and reduce the time for analysis, thus, contributing substantially towards the development of future pharmacological agents for the treatment of DR.
\end{abstract}

Keywords: Diabetic Retinopathy, Acellular capillaries, Segmentation, Quantification, Capillary Detection, Medial Axis Transform

\section{INTRODUCTION}

Diabetic retinopathy (DR) is a long-term complication of diabetes and the majority of patients during the first twenty years of the disease will develop some form of DR. ${ }^{1}$ The pathogenic nature of the diabetic milieu causes a progressive loss of retinal capillary cells (pericytes and endothelial cells), leaving non-perfused acellular capillaries. $^{2}$ In animal models, the number of acellular capillaries is a marker of DR severity and response to the treatment. Traditionally, the acellular capillaries are counted in a single-blind fashion by more than one independent investigator, either directly through a microscope or via manual counting of captured images. However, this system is laborious, time-consuming, and often shows significant inter-observer variability. Some studies in the past have attempted to characterize the retinal vasculature in terms of retinal vessel caliber, blood vessel tortuosity ${ }^{3}$ and, vessel pattern in humans ${ }^{4}$ and in animal models; ${ }^{5}$ however, there is a dearth of automated software platforms. Therefore, the purpose of this study was to develop a computer-based algorithm for the quantification of acellular capillaries, the gold standard for the assessment of experimental DR.

Further author information: (Send correspondence to Ashay D. Bhatwadekar)

Ashay D. Bhatwadekar: E-mail: abhatwad@iupui.edu

Medical Imaging 2020: Biomedical Applications in Molecular, Structural, and Functional Imaging, edited by Andrzej Krol, Barjor S. Gimi, Proc. of SPIE Vol. 11317, 113170N · C 2020 SPIE · CCC code: 1605-7422/20/\$21 · doi: 10.1117/12.2543400 


\section{METHODS}

\subsection{Animal studies}

All animal studies obtained prior approval by the institutional animal care and use committee. Studies were performed as per the guidelines of The Guiding Principles in the Care and Use of Animals (NIH) and conformed to the Association for Research in Vision and Ophthalmology (ARVO) statement for the use of animals in ophthalmic and vision research. The wild-type (WT; C57BL/6J) mice at about 4 weeks of age were purchased from the Jackson Laboratories (Bar Harbor, ME). (i) Induction of diabetes: Streptozotocin (IP at a dose of 40 $\mathrm{mg} / \mathrm{kg}$ in citrate buffer ( $\mathrm{pH} 4.5$ ) on 5 consecutive days) was used to induce diabetes in WT mice, while another group received the vehicle and served as a control. The animals were euthanized after 6 months and processed for the trypsin digestion as described below. (ii) Trypsin digestion of retina: The retinas were trypsin digested as described previously. ${ }^{6}$ The slides were stained with periodic acid-Schiff's (PAS) followed by counterstaining with hematoxylin (Sigma-Aldrich St. Louis, MO). Acellular capillaries were counted at 20X magnification in eight different fields.

\subsection{Acellular capillary detection and counts}

Our program provides a direct count of acellular capillaries within minutes. This program accepts high-quality images in .tiff and .jpeg formats and a minimum recommended resolution is $400 \times 400$ for efficient detection of acellular capillaries. The program is designed to scale every image to $1388 \times 1041$ pixels before it processes them. The program uses well-established low-level image processing and morphological operators for the detection of the acellular capillaries. The novelty of our program is in the application of the above methods to the domain of counting acellular capillaries for which there are not many known automated programs. The following are overall steps (i) preprocessing for image enhancement and noise reduction, (ii) automatic determination of the thresholds for segmenting the foreground/background regions, (iii) determining the medial axes of the foreground (capillaries), and (iv) for a given capillary, determining the thickness by taking in account the distance of boundaries to the medial axis, and thresholding based on this thickness. The program is designed to identify acellular capillaries regardless of the image source (bright field or fluorescent image). Therefore, the input image (Figure 1A) was first converted to grayscale. In order to make the image segmentation insensitive to slowly varying background lighting, the image was smoothed using a Gaussian filter followed by a background subtraction $^{7}$ (Figure 1B). This was done by a morphological closing operator using a disk structuring element with a radius of 128 pixels. As a result of this, the image has uniform background bright regions, with the capillaries making up the darker foreground regions, thus making the segmentation task easier. The darker regions making up the foreground consist of a lighter shade of gray (capillaries) and darker shade of gray (cells). Thus, the image has a trimodal histogram. Therefore, we used a mixture of three Gaussians to fit to the histogram in order to obtain our thresholds to be used for segmentation. The fitting was performed using Expectation-Maximization (EM) algorithm as implemented in Python Numpy. ${ }^{8}$ The three modes correspond to (a) one mode as bright background and two modes as two shades as foreground: (b) capillaries and (c) cell nuclei. The threshold was determined adaptively by identifying the lowest point in the valley between the background mode (brighter pixels) and the next foreground mode representing the capillaries. This threshold was used to generate a binary image with capillaries as foreground (Figure 1C). Post-processing was done on the segmented image to eliminate 'salt-and-pepper noise' and 'small holes' in the foreground using morphological operators of opening/closing. ${ }^{9}$ On the resulting image, the Medial Axis Transformation (MAT) ${ }^{10}$ was computed for the foreground regions (capillaries) to (a) skeletonize and (b) to determine the distance of each point on the medial axis to the vessel boundary (Figure 1D). Using this MAT representation, the candidate acellular capillary branches were isolated at branch points (Figure 1E). For each capillary segment, a threshold was used to determine if it is acellular or not as follows: (a) the median thickness was computed for each candidate acellular capillary along its MAT curve, and (b) the median of these median thicknesses was computed. (c) The threshold for deciding an acellular capillary then was determined adaptively based on the magnification factor. For the images taken at the 20X magnification, a threshold was selected as half the median thickness, while for the images taken at the 40X the threshold was selected as a quarter of median thickness. The threshold value was determined as a value greater than the median length of all acellular capillaries. Finally, a connected component analysis was done on the detected capillaries to obtain a count of the number of acellular capillaries in the image.

Proc. of SPIE Vol. 11317 113170N-2 

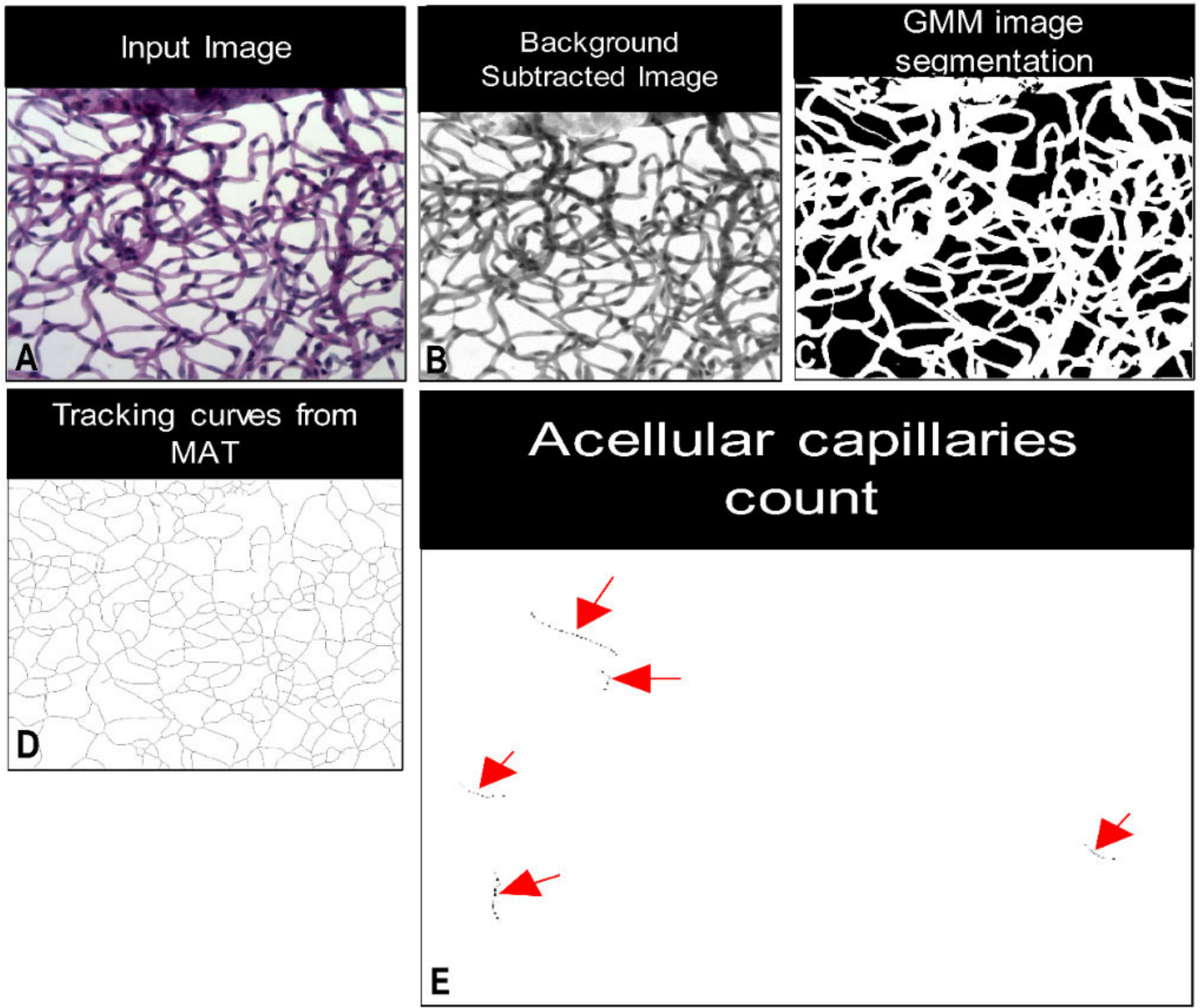

Figure 1. Sequential segmentation of retinal trypsin digests for enumeration of acellular capillaries. Representative images of trypsin digest showing steps taken for enumeration of acellular capillaries. (A) An input image was scaled to the program (B) followed by a background subtraction, (C) a Gaussian mixture model (GMM) based image segmentation and (D) medial axis transformation (MAT). (E) The MAT was tracked for curves to finally identify the acellular capillaries (red arrows).

\subsection{Statistics}

The enumeration of acellular capillaries was performed by three independent blinded investigators in parallel with the computerized assessment. The data in bar charts are represented as mean $\pm \mathrm{SEM}$. The statistical analysis was performed using one-way ANOVA followed by posthoc Student's Newman Keul Test using GraphPad Prism Software (La Jolla, CA, USA). The mean difference of $p<0.05$ was considered to be statistically significant.

\section{RESULTS}

\subsection{Identification of acellular capillaries at different magnifications}

We first determined the consistency of our program in the identification of acellular capillaries in trypsin digest images of the same retinal region at 20X and 40X magnification (Figure 2A). The program efficiently detected 
acellular capillaries at both magnifications and the difference in counts was statistically insignificant (Figure 2B). Therefore, all further assessments were performed on images taken at 20X magnification only.

A

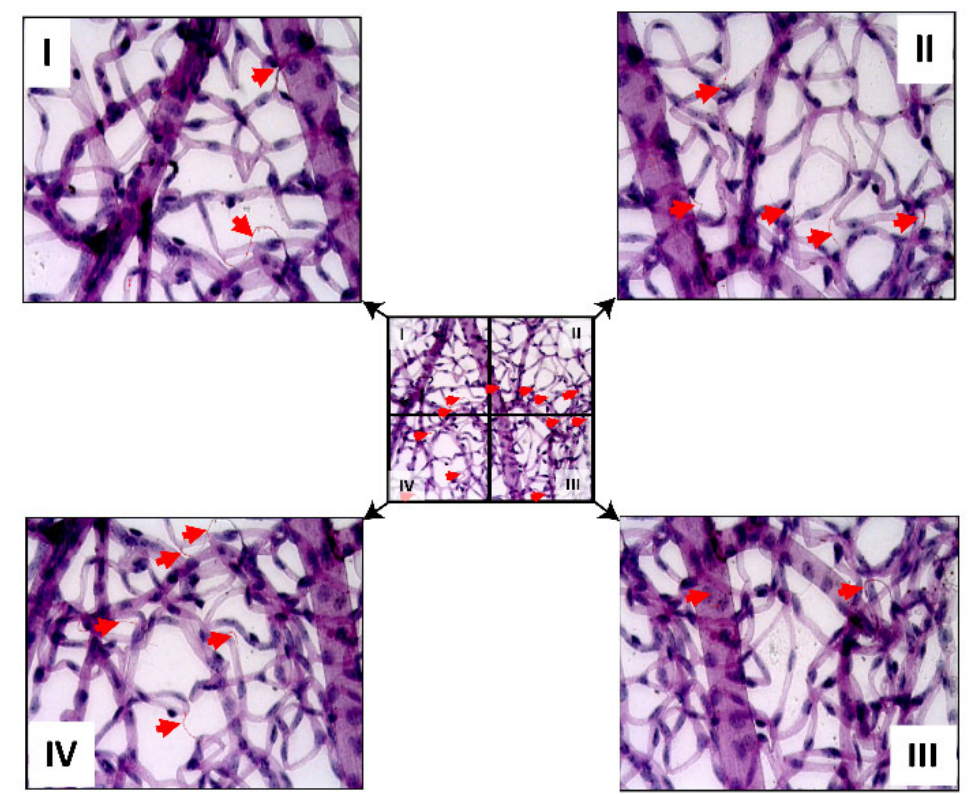

B

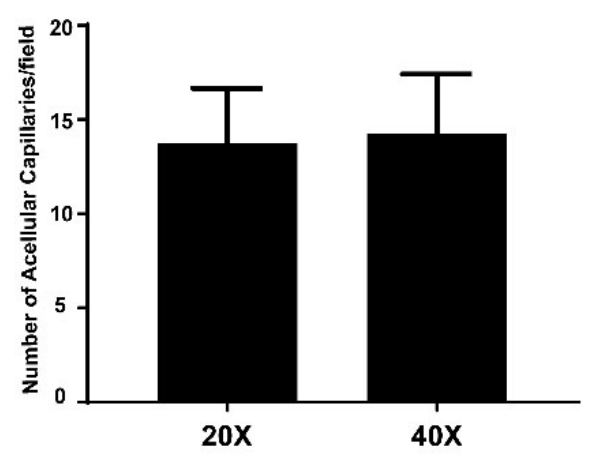

Figure 2. Enumeration of acellular capillaries at different magnifications. (A) A representative image of retinal trypsin digest taken at both $20 \mathrm{X}$ and 40X magnification. (B) Bar chart showing quantification of acellular capillaries, $n=4$.

\subsection{Enhanced consistency in retinopathy assessment}

We evaluated the number of acellular capillaries in an animal model of diabetes. The images were counted by both independent investigators and using our image analysis platform. The enumeration of acellular capillaries by three blinded investigators revealed significant variation in counts between investigators $\mathrm{A}$ and $\mathrm{C}$ (investigator effect, $p<0.01$; two-way ANOVA). Overall, the diabetic mice showed an increase in acellular capillaries in comparison to WT mice (Figure 3A). The acellular capillary number was 1.6-fold greater $(p<0.001)$ in diabetic retinas compared to WT when assessed by manual counting and 1.5 -fold greater $(p<0.01)$ when computed automatically; the difference was not statistically significant (Figure 3B). 

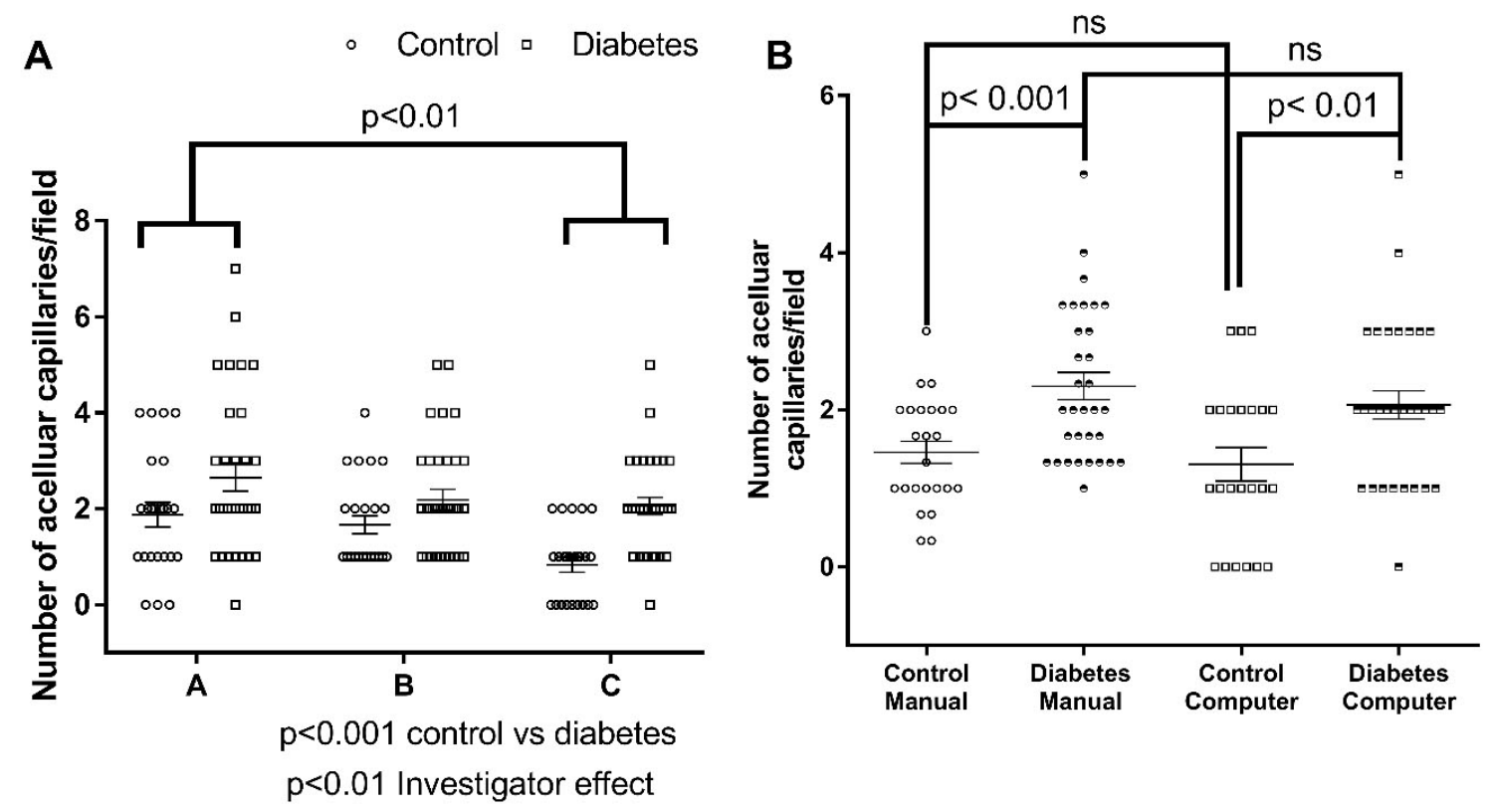

Figure 3. Computer-based identification of acellular capillaries in a mouse model of type 1 diabetes. The acellular capillaries were enumerated using a computerized algorithm and manual counting. (A) Chart showing counts by blinded investigators A, B, and C, (B) chart showing a comparison of acellular capillary counts of control and treatment groups between manual and computer counts in type 1 diabetes, $n=6$ control; $n=3$ diabetes.

\section{CONCLUSIONS}

DR is the most prevalent complication of diabetes, and the development of pharmacological agents is important for tackling this vision-threatening eye disease. Enumeration of acellular capillaries is routinely used as a marker for a response to the pharmacological intervention. We developed a sensitive, fast and user-friendly tool for quantification of acellular capillaries in DR. This computer program successfully identified acellular capillaries in an animal model of T1D. The image analysis program was tested on a mouse retina using optimum threshold intensities at different magnifications. Initial studies were performed on a set of images to identify the threshold intensity and a generalized branch point detection algorithm that will accommodate each image given as an input.

This program works efficiently on both mouse and rat retinas at both 20X and 40X magnifications. The image quality is one of the most important factors for reducing the false positive numbers. Specifically, we recommend high-quality images (tiff or jpeg formats with minimal or no compression) with optimum white balance for the enumeration of the acellular capillaries. Some of the greatest challenges we faced involved background noise in the backlit regions of the image, histogram-based threshold determination and tracking the medial axis curve. One of the limitations of the program is its inability to process images that are of low quality, with a darker background that contains a lot of noise; however, brightfield images from retinal digests tend to be of high quality with little background material. We used well-established low-level image-processing methods such as thresholding, morphological operations, and MAT computation for identifying the capillaries in our program. Other programs/methods such as the use of deformable models have also been used in some studies e.g., in the three-dimensional tracing of neurites. ${ }^{11,12}$ We speculate that these methods would be equally competent for image analysis of acellular capillaries, however, these methods are much more computationally intensive than the one described in this manuscript.

In conclusion, our study has developed an automated tool for characterization of retinopathy which is fast with good performance in the quantification of acellular capillaries. This sensitive, user-friendly and freely available tool can be used successfully for evaluating the response of pharmacological treatment for protection from retinopathy. 


\section{ACKNOWLEDGMENTS}

This work is supported by the funding from the Center of Diabetes \& Metabolic Diseases, Indiana University's Pilot \& Feasibility Award and from the National Institute of Health-National Eye Institute's award, EY027779 to $\mathrm{AB}$.

\section{REFERENCES}

[1] Fong, D. S., Aiello, L., Gardner, T. W., King, G. L., Blankenship, G., Cavallerano, J. D., Ferris, F. L., and Klein, R., "Diabetic retinopathy," Diabetes Care 26(suppl 1), s99-s102 (2003).

[2] Hammes, H.-P., Lin, J., Renner, O., Shani, M., Lundqvist, A., Betsholtz, C., Brownlee, M., and Deutsch, U., "Pericytes and the pathogenesis of diabetic retinopathy," Diabetes 51(10), 3107-3112 (2002).

[3] Wallace, D. K., "Computer-assisted quantification of vascular tortuosity in retinopathy of prematurity (an american ophthalmological society thesis)," Transactions of the American Ophthalmological Society 105, 594-615 (2007).

[4] Trucco, E., Ballerini, L., Relan, D., Giachetti, A., MacGillivray, T., Zutis, K., Lupascu, C., Tegolo, D., Pellegrini, E., Robertson, G., Wilson, P. J., Doney, A., and Dhillon, B., "Novel vampire algorithms for quantitative analysis of the retinal vasculature," in [2013 ISSNIP Biosignals and Biorobotics Conference: Biosignals and Robotics for Better and Safer Living (BRC)], 1-4 (Feb 2013).

[5] Ghanian, Z., Staniszewski, K., Jamali, N., Sepehr, R., Wang, S., Sorenson, C. M., Sheibani, N., and Ranji, M., "Quantitative assessment of retinopathy using multi-parameter image analysis," Journal of medical signals and sensors 6(2), 71-80 (2016).

[6] Bhatwadekar, A., Glenn, J. V., Figarola, J. L., Scott, S., Gardiner, T. A., Rahbar, S., and Stitt, A. W., "A new advanced glycation inhibitor, LR-90, prevents experimental diabetic retinopathy in rats," British Journal of Ophthalmology 92(4), 545-547 (2008).

[7] Parks, D. H. and Fels, S. S., "Evaluation of background subtraction algorithms with post-processing," in [2008 IEEE Fifth International Conference on Advanced Video and Signal Based Surveillance], 192-199 (Sep. 2008).

[8] Dempster, A. P., Laird, N. M., and Rubin, D. B., "Maximum likelihood from incomplete data via the em algorithm," Journal of the Royal Statistical Society. Series B (Methodological) 39(1), 1-38 (1977).

[9] Barrera, J., d. A. Zampirolli, F., and d. A. Lotufo, R., "Morphological operators characterized by neighborhood graphs," in [Proceedings X Brazilian Symposium on Computer Graphics and Image Processing], 179-186 (Oct 1997).

[10] Lee, D. T., "Medial axis transformation of a planar shape," IEEE Transactions on Pattern Analysis and Machine Intelligence PAMI-4, 363-369 (July 1982).

[11] Gulyanon, S., Sharifai, N., Kim, M. D., Chiba, A., and Tsechpenakis, G., "CRF formulation of active contour population for efficient three-dimensional neurite tracing," in [13th IEEE International Symposium on Biomedical Imaging, ISBI 2016, Prague, Czech Republic, April 13-16, 2016], 593-597, IEEE (2016).

[12] Gulyanon, S., Sharifai, N., Bleykhman, S., Kelly, E., Kim, M. D., Chiba, A., and Tsechpenakis, G., "Threedimensional neurite tracing under globally varying contrast," in [12th IEEE International Symposium on Biomedical Imaging, ISBI 2015, Brooklyn, NY, USA, April 16-19, 2015], 875-879, IEEE (2015). 